\title{
ADAM10 hyperactivation acts on piccolo to deplete synaptic vesicle stores in Huntington's disease
}

Flora Cozzolino ${ }^{1,2 \#}$, Elena Vezzoli ${ }^{3 \#}$, Cristina Cheroni ${ }^{4}$, Dario Besusso ${ }^{5,6}$, Paola Conforti ${ }^{5,6}$, Marta Valenza ${ }^{5,6}$, Ilaria Iacobucci ${ }^{1,2}$, Vittoria Monaco ${ }^{2,7}$, Giulia Birolini ${ }^{5,6}$, Mauro Bombaci ${ }^{6}$, Andrea Falqui ${ }^{8 \S}$, Paul Saftig ${ }^{9}$, Riccardo L. Rossi ${ }^{6}$, Maria Monti ${ }^{1,2}$, Elena Cattaneo ${ }^{5,6}$, Chiara Zuccato ${ }^{5,6^{*}}$

${ }^{1}$ Department of Chemical Sciences, University of Naples "Federico II", 80126 Naples, Italy.

${ }^{2}$ CEINGE Advanced Biotechnologies, 80131 Naples, Italy.

${ }^{3}$ Department of Biomedical Sciences for Health, University of Milan, 20133 Milan, Italy.

${ }^{4}$ European Institute of Oncology, IRCCS, 20141 Milan, Italy; Department of Oncology and Hemato-oncology, University of Milan, 20122 Milan, Italy.

${ }^{5}$ Department of Biosciences, University of Milan, 20133 Milan, Italy.

${ }^{6}$ Istituto Nazionale di Genetica Molecolare "Romeo ed Enrica Invernizzi", 20122 Milan, Italy.

${ }^{7}$ Biostructures and Biosystems National Institute (INBB), 00136 Rome, Italy.

${ }^{8}$ King Abdullah University of Science and Technology (KAUST), Biological and Environmental Science \& Engineering (BESE) Division, NABLA Lab, 23955 Thuwal, Saudi Arabia.

${ }^{9}$ Institute of Biochemistry, Christian-Albrechts-University of Kiel, D-24098 Kiel, Germany.

$\S$ present address Department of Physios, University of Milan, 20133 Milan, Italy.

${ }^{\#} \mathrm{FC}$ and $\mathrm{EV}$ contributed equally to this work.

(C) The Author(s) 2021. Published by Oxford University Press. All rights reserved. For Permissions, please email: journals.permissions@oup.com 
*To whom correspondence should be addressed at: Department of Biosciences, University of Milan, 20133 Milan, Italy; Istituto Nazionale di Genetica Molecolare "Romeo ed Enrica Invernizzi," 20122 Milan, Italy. Tel: +39 02 50325839; Fax: +3902 50325843; Email: chiara.zuccato@unimi.it 


\section{Abstract}

Synaptic dysfunction and cognitive decline in Huntington's disease (HD) involve hyperactive A disintegrin and metalloproteinase domain-containing protein 10 (ADAM10). To identify the molecular mechanisms through which ADAM10 is associated with synaptic dysfunction in HD, we performed an immunoaffinity purificationmass spectrometry (IP-MS) study of endogenous ADAM10 in the brains of wild-type and HD mice. In the normal brain, proteins implicated in synapse organization, synaptic plasticity, and vesicle and organelles trafficking interact with ADAM10, suggesting that it may act as a hub protein at the excitatory synapse. Importantly, the ADAM10 interactome is enriched in presynaptic proteins and ADAM10 coimmunoprecipitates with piccolo (PCLO), a key player in the recycling and maintenance of synaptic vesicles (SVs). In contrast, reduced ADAM10/PCLO immunoprecipitation occurs in the HD brain, with decreased density of SVs in the reserve and docked pool at the HD presynaptic terminal. Conditional heterozygous deletion of ADAM10 in the forebrain of HD mice reduces active ADAM10 to wild-type level, and normalizes ADAM10/PCLO complex formation and SVs density and distribution. The results indicate that presynaptic ADAM10 and PCLO are a relevant component of HD pathogenesis. 


\section{Introduction}

A disintegrin and metalloproteinase domain-containing protein 10 (ADAM10) is one of the 22 members of the ADAM family of transmembrane proteases found in humans, and one of the most widely expressed in the brain (1). Nascent ADAM10 is produced as an approximately $95 \mathrm{kDa}$ zymogen that is converted into its proteolytically active form, called mature ADAM10 (m-ADAM10, $60 \mathrm{kDa}$ ), after cleavage of its prodomain by furin or proconvertase 7 (PC7) in the trans-Golgi secretory pathway (1). Within neurons, ADAM10 localizes specifically at the synapse and is associated with the postsynaptic compartment, together with the scaffold protein synapseassociated protein 97 (SAP97) (2) and clathrin adaptor AP2 (3), which are required, respectively, for insertion of ADAM10 into the synaptic membrane and for its endocytosis. The attractiveness of this enzyme is that it is the main physiological alpha-secretase in neurons responsible for non-amyloidogenic cleavage of amyloid precursor protein (APP) (1). Moreover, ADAM10 has a large number of synaptic substrates, indicating extensive flexibility of the protease with regard to substrate recognition and cleavage in the brain (4). Consequently, the biological effect of ADAM10 activity may change over time and in different conditions, as it relies on the presence and function of the target substrates and cleavage products. Accordingly, ADAM10 plays fundamental roles during brain development and in the homeostasis of adult neuronal networks $(1,5)$, as it is involved in dendritic spine formation, maturation, and stabilization as well as in axon guidance and extension, in neuronal migration and myelination and also in the molecular organization of the glutamatergic synapse (1,5). ADAM10-dependent cleavage of N-cadherin (N-CAD), nectin cell adhesion molecule 1 (NECTIN1), and Neuroligin 1 (NLGN1) also represents a new modality for regulating the strength and activity of the glutamatergic synapse $(1,5)$. An excess of ADAM10-mediated N-CAD proteolysis in the brain has been reported to compromise adhesion between the presynaptic and postsynaptic membrane and reduces the overall long-term stability and neurotransmission of the glutamatergic synapse (6-8).

Defects in this circuitry have been implicated in Huntington's disease (HD), an autosomal dominant neurodegenerative disorder caused by an unstable CAG triplet repeat expansion in exon 1 of the huntingtin gene (HTT) (9). An alteration in presynaptic and/or postsynaptic compartments has been demonstrated in several mouse models of the disease since the early stages and documented in HD patients in cross-sectional and neuroimaging studies (10-12).

ADAM10 has been linked to HTT and dysfunction of the glutamatergic synapse in HD. Studying neurulation in embryonic stem cells depleted of HTT, we found that the homotypic interaction between neuroepithelial cells is 
dependent on the presence of normal HTT, which acts physiologically to inhibit ADAM10 and the shedding of its substrate N-CAD allowing cell-cell contacts (13). We also reported that, in the adult brain, normal HTT similarly affects synapse remodeling through inhibition of ADAM10 activity on N-CAD (13). In HD, this pathway is hyperactive, with deleterious consequences on the morphology and functionality of the glutamatergic synapse (14). In particular, we found that ADAM10 accumulates at postsynaptic density (PSD) and causes excessive NCAD proteolysis (14), leading to loss of excitatory synaptic contacts, synapse deficiencies, and cognitive deeline in HD mice. We also demonstrated that pharmacological, molecular, and genetic approaches aimed at normalizing the level of the active enzyme in the HD mouse brain prevent electrophysiological synaptic defects at the excitatory synapse and cognitive impairment in the mice (14). Taken together, these previous studies point to a critical role of ADAM10 at the glutamatergic synapse in the HD brain.

To unravel the molecular mechanisms through which ADAM10 is associated with glutamatergic synaptic dysfunction in HD, we performed a system-level study of ADAM10 interactors in the brain of HD mice. A functional proteomic experiment based on immunoaffinity purification-mass spectrometry (IP-MS) identification of endogenous ADAM10 interactors in the brains of wild-type and HD mice revealed molecular targets that link ADAM10 to the normal and HD glutamatergic synapse. This study also identifies piccolo (PCLO) as an ADAM10 partner, suggesting a new role of the enzyme at the presynaptic terminal. Furthermore, we show that, in the HD mouse brain in vivo, mutant HTT causes presynaptic defects by affecting the formation of the ADAM10/PCLO complex and, consequently, the synaptic vesicles (SVs) distribution in the presynaptic bouton.

\section{Results}

\section{The ADAM10 interactome is enriched in synaptic proteins}

We established the interactome of ADAM10 in the striatum of 50-week-old wild-type mice and compared it to the interactome of homozygous HD (zQ175) knock-in mice (15) of the same age. We adopted a proteomic approach consisting of the isolation of ADAM10 protein complexes by immunoprecipitation, followed by protein identification by mass spectrometry.

Fig. 1A shows the workflow for the entire procedure. Following tissue collection, we prepared protein tissue lysates from four mice/genotype and performed Western blot for ADAM10. We confirmed that the level of mADAM10 is increased in the zQ175 striatum compared to the wild-type control (Fig. 1B,C). Tissue lysates from 
each genotype were then pooled and subjected to ADAM10 immunoprecipitation following a pre-clearing step on Dynabeads-Protein G. For ADAM10 immunoprecipitation, we used an in-house rabbit polyclonal antibody that recognizes the C-terminal amino acid residues of ADAM10 and is able to detect both the zymogen $(110 \mathrm{kDa})$ and the active forms of the enzyme (m-ADAM10, $60 \mathrm{kDa}$ ). The antibody has been extensively validated in the past by Western blot of brain tissue lysates prepared from wild-type and CaMKII $\alpha$-Cre:Adam10 ${ }^{\text {Flox/Flox }}$ mice $(14,16)$ and successfully used in co-immunoprecipitation (co-IP) experiments with mouse brain samples (17). Proteins retained by Dynabeads-Protein G during pre-clearing of wild-type and zQ175 tissue lysates in the absence of specific antibodies were eluted and used as controls; both samples and the pre-clearing controls were fractionated by SDS-PAGE and the lanes cut into 33 gel bands, which were digested in situ with trypsin. The resulting peptide mixtures were directly analyzed by nanoscale liquid chromatography coupled to tandem mass spectrometry (nanoLC-MS/MS). Mass spectral data were used to search a non-redundant protein database using Mascot software. Proteins detected in the samples and in the corresponding pre-clearing controls were discarded, as they represented proteins binding non-specifically to the beads, and only those solely present in the sample lanes were considered as candidates for ADAM10 interactors.

Two lists of putative ADAM10 interactors were obtained, one in the wild-type striatum and another in the zQ175 striatum (Supplementary Material, Table S1). To filter out potential contaminants, we relied on the Contaminant Repository for Affinity Purification database, a web-accessible resource that stores and annotates negative control experiments generated by the proteomics research community (18). Considering non-specific contaminant proteins reported in at least $50 \%$ of the experiments collected by the database, we excluded 25 proteins from downstream analyses. Thus, a final list of 197 putative ADAM10 interactors are included in Supplementary Material, Table S2. These putative interactors are visualized in Fig. 1D as a network. Ninetyone interactors are shared among the two conditions, whereas 68 and 38 are found selectively in the wild-type or mutant brain, respectively.

Next, we characterized the putative ADAM10 interactors by Gene Ontology enrichment analysis. We relied on the striatal proteome of zQ175 mice (19) as a background of expressed proteins against which the interactors have been compared for the enrichment analysis. By interrogating the Cellular Component domain and imposing the thresholds $\mathrm{P}<0.01$ and enrichment $\geq 2$, we identified 38 enriched ontology categories in wild-type and 34 in zQ175. Focusing on the top-12 categories (ranked by P value, Fig. 1E), most were shared between the two conditions, with similar enrichment and P values. Notably, this holds true for the top-three terms: glutamatergic 
synapse, PSD, and presynaptic active zone (AZ) (Fig. 1E and Supplementary Material, Fig. S1). Of particular interest was the presence of presynaptic proteins of the AZ, a specialized area of the presynaptic bouton composed of sophisticated protein machinery implicated in SVs fusion (20). Though the postsynaptic role of ADAM10 is widely recognized, the presynaptic function(s) of this protease has not been described and its association with the presynapse is a novel finding.

We also evaluated whether the mRNA levels of ADAM10 interactors can be modulated by mutant HTT. We interrogated the published results for the differential expression analysis performed by comparing the striatum of 10-month-old zQ175 mice to zQ20 mice (19) and retrieved the fold-change (FC) and false discovery rate (FDR) for each ADAM10 interactor. The dataset was then overlaid with the ADAM10 interactome network, visualizing proteins with an FDR $<0.1$ and the directionality of the FC based on the node color (Supplementary Material, Fig. S2A). Overall, ADAM10 interactors were not strongly modulated by mutant HTT at the transcriptional level, with even modest FCs for significant modulations (Supplementary Material, Fig. S2B). This analysis excluded the possibility that the mRNA level of presynaptic ADAM10 interactor candidates could be modulated by the presence of an expanded polyQ tract in HTT.

Given the deleterious association of ADAM10 with HTT previously identified in HD models $(13,14)$, we also evaluated whether any previously reported HTT-interacting proteins were present in our network. We interrogated the HTT interactome curated in the HDinHD database (www.hdinhd.org), extracting 1625 mouse and human HTT interactors for a comparison with our dataset. Overall, 105 ADAM10 interactors were shared with HTT, representing more than 50\% of our reconstructed ADAM10 interactome (Fig. 2A). Gene Ontology analyses of this subset confirmed once again the emergence of terms related to the glutamatergic synapse, presynaptic zone, and PSD as the top-three categories (Fig. 2B). Interestingly, we found that ADAM10 shares protein partners with HTT at the presynaptic terminal, including such critical molecules of the AZ, as bassoon (BSN), PCLO, ELKS/RAB6-Interacting/CAST Family Member 2 (ERC2), and liprin-alpha-3 (PPFIA3) (Fig. 2C). We concluded that the brain ADAM10 interactome is enriched with proteins of the glutamatergic synapse, and new unexpected presynaptic proteins emerge among candidate ADAM10 interactors, suggesting a novel function of the protease at the presynaptic bouton. As ADAM10 and HTT share presynaptic proteins as putative interactors, we speculated that ADAM10 may be active at the presynapse in the organization and dynamics of the SVs pools, and that this function is affected in HD. 


\section{Active ADAM10 is increased at the presynaptic terminal in HD}

Since the presence of ADAM10 at the presynaptic terminal has not been described before, we started by evaluating the level of ADAM10 protein in the presynaptic compartment of wild-type and HD mice. For these studies we selected the widely used R6/2 mouse model, which bears a genomic fragment containing the promoter and exon 1 of human HTT with 144 CAGs (21) and exhibits an increased level of active ADAM10 in the striatum and cortex (14). We prepared synaptosomes, presynaptic and postsynaptic fractions from the cortical tissues of 12-week-old wild-type and R6/2 mice (Fig. 3A). We specifically focused on the cortex because cortical projection neurons represent the main presynaptic input to the striatum, the brain area that predominantly degenerates in HD (22). The level of m-ADAM10 was increased up to 2.5 -fold in cortical synaptosomes from R6/2 mice compared to wild-type mice (Fig. 3B,C). After confirming the correct ultrastructural fractionation by immunoblotting for synaptophysin (SYP) and postsynaptic density protein 95 (PSD-95), which identifies the presynaptic and postsynaptic compartment, respectively, we performed immunoblotting with an anti-ADAM10 antibody. The level of the active form of the enzyme was increased up to 2.6-fold in cortical postsynaptic fractions from R6/2 mice compared to wild-type littermates, although levels of PSD-95 was reduced as previously described (23) (Fig. 3B,C). Notably, active ADAM10 was also present in the cortical presynaptic terminal, and its level increased up to 2.2 -fold in R6/2 mice compared to the wild-type mice (Fig. 3B,C).

\section{Active ADAM10 and the presynaptic protein PCLO form a complex that is affected in the HD brain}

To validate the predicted interaction of ADAM10 with proteins of the AZ, we performed co-IP experiments on lysates from the cortices of 12-week-old wild-type and R6/2 mice. We focused on PCLO because of its recognized role in the structural and functional assembly of presynaptic AZ, in guiding SVs from the backfield of the synapse to the AZ for release, and in the recycling and maintenance of SVs $(24,25)$. We found that the active form of ADAM10 co-immunoprecipitated with PCLO in total protein lysates prepared from the wild-type mouse cortex (Fig. 3D). Interestingly, binding of the catalytically active form of ADAM10 to PCLO was decreased 50\% in the cortices of symptomatic 12 week-old R6/2 mice (Fig. 3D,E), though the level of active ADAM10 was increased in the input samples (14) (Fig. 3D,E). Since PCLO levels, normalized either against $\alpha$-tubulin or RNA polymerase II, were similar in cortices from 12-week-old wild-type and R6/2 transgenic mice (Fig. 3D,E;

Supplementary Material, Fig. S3), we excluded the possibility that the observed quantitative changes in interaction were due to changes in PCLO protein level in HD. 
We also evaluated whether normalizing the ADAM10 level in the R6/2 forebrain would prevent reduced formation of the ADAM10/PCLO complex. We crossed R6/2 mice with CaMKII $\alpha$-Cre:Adam10 ${ }^{\text {Flox/t }}$ mice (A10cKO), an ADAM10 heterozygous conditional knock-out mouse line limiting ADAM10 inactivation to the postnatal forebrain (16), to obtain R6/2-A10cKO mice (Supplementary Material, Fig. S4A). Western blotting of R6/2-A10cKO cortical synaptosomes showed that the levels of m-ADAM10 were restored to wild-type levels (Supplementary Material, Fig. S4B,C). Co-IP showed the formation of a complex between m-ADAM10 and PCLO in cortical synaptic fractions from 12-week-old wild-type mice (Fig. 3F). Moreover, we found that the binding of the catalytically active form of ADAM10 to PCLO was reduced 76\% in the cortical synaptosomes of symptomatic 12-week-old R6/2 mice, though the level of active ADAM10 was increased (Fig. 3F and

Supplementary Material, Fig. S4). These data confirmed and extended previous data obtained on total cortical tissue lysates from 12-week-old wild-type and R6/2 mice by showing that co-IP between m-ADAM10 and PCLO is reduced in the presence of mutant HTT (Fig. 3D,E). Notably, formation of the ADAM10/ PCLO complex in R6/2-A10cKO mice was almost restored to the level of wild-type mice (Fig. 3F; Supplementary Material, Fig. S5).

Taken together, these findings indicate that active ADAM10 forms a complex with presynaptic protein PCLO. In HD, when the level of m-ADAM10 increases, the binding of the protease to PCLO is reduced.

\section{Normalizing ADAM10 prevents presynaptic defects in the $\mathrm{R} 6 / 2$ cortex}

As PCLO is implicated in regulating SVs density, as well as the dynamics and distribution of SVs (24-27), we used transmission electron microscopy (TEM) to test SVs density and distribution in both R6/2 and R6/2A10cKO cortical neurons. TEM analyses revealed a $25 \%$ reduction in vesicle density in R6/2 cortical presynaptic boutons with respect to wild-type mice (Fig. 4B,C) with similar synaptic area, AZ length, and vesicle diameter (Fig. 4D-F). Next, we compared the cumulative \% of SVs distribution within 0 to $500 \mathrm{~nm}$ from the AZ in the wild-type and R6/2 presynaptic terminal. In R6/2 mice, the cumulative \% of SVs distribution had a markedly disturbed profile (Fig. 4G). Based on distance-to-membrane measurements, vesicles are distributed into three different pools (docked, 0-50 nm; reserve, 50-300 nm; resting, >300 nm) (Fig. 4A). The density of SVs in the docked and reserve pools was reduced in R6/2 mice compared to wild-type, but we found no differences among genotypes in the density of SVs in the resting zone (Fig. 4H-J). We also evaluated whether normalization of ADAM10 can rescue presynaptic vesicle defects in R6/2 cortical neurons in vivo. We found that the cumulative \% 
of SVs distribution in R6/2-A10cKO presynaptic terminal was similar to the wild-type mice. Reduction in the total SVs density was also completely restored in R6/2-A10cKO cortical synapses (Fig. 4C), and synaptic vesicle numbers was increased in the docked and reserve pools of R6/2-A10cKO mice to the same levels as the wild-type group (Fig. 4H, I). No differences in presynaptic parameters were found in the cortices of A10cKO mice, confirming that heterozygous suppression of the ADAM10 gene in the healthy mouse forebrain had no effect on synapse morphology (Fig. 4C-J) (14). Furthermore, we did not find differences in PSD length between the analyzed genotypes (Supplementary Material, Fig. S6).

Taken together these data indicate for the first time the presence of presynaptic morphological defects in HD cortical neurons which are associated with ADAM10 dysfunction. Specifically, SVs density is reduced in the cerebral cortex in HD, and depleted SVs stores are restricted to presynaptic regions with a higher turnover and actively engaged in release and recycling. Moreover, we show that genetic reduction of ADAM10 levels restored physiological SVs density and distribution in HD mice.

\section{Discussion}

Hyperactive ADAM10 has been linked to synaptic dysfunction and cognitive decline in HD (14). To identify which ADAM10 interactors may participate in this synaptic dysfunction, we performed the first IP-MS study of endogenous ADAM10 in the brain using wild-type and HD mice. In addition to revealing known ADAM10interacting proteins implicated in ADAM10 trafficking and endocytosis, such SAP97 (2), tetraspanin-15 (TSPAN15) (17), and adaptor related protein complex 2 subunit alpha 1 (AP2A1) (3) (Fig. 1D), we identified molecules of the glutamatergic synapse, including proteins of the PSD and presynaptic AZ, as the top-three ontological terms of ADAM10 interactors. Moreover, among the ADAM10 interactors, we found proteins implicatéd in synaptic plasticity and vesicle trafficking. Interestingly, by integrating our ADAM10 dataset with the available HTT interactome from HDinHD (https://hint.hdinhd.org/interactions), we were able to demonstrate that the resulting proteins were significantly enriched with components of the presynaptic machinery, suggesting a presynaptic function of ADAM10, which is disrupted in HD.

Specifically, we show that the active form of ADAM10 is present in presynaptic fractions and that it binds to PCLO, the largest multidomain protein of the AZ with a structural role in SVs organization (24-27). PCLO exerts multiple functions at the $\mathrm{AZ}$ and is implicated in endosome formation and the maintenance of the SVs reserve 
pool (25). PCLO also regulates the translocation of SVs from the reserve to the ready releasable pool (26) and safeguards a fraction of them for delayed action potential-induced release. This enables the synapse to sustain high frequency synaptic transmission over long periods (27). Our work indicates that ADAM10, through its binding to PCLO, is involved in presynaptic functions, specifically in the regulation of SVs dynamics at the AZ. We also demonstrated the presence of morphological presynaptic defects, particularly an altered distribution of SVs at the AZ of neurons of the HD cortex, which correlate with reduced formation of the m-ADAM10 PCLO complex. These findings indicate that ADAM10 and PCLO at the presynapse can be a relevant component of HD pathogenesis and reinforces the role of the cortex and the presynaptic compartment in $\mathrm{HD}$, which have to be considered in future therapies.

This study suggests a novel aspect of ADAM10 biology and creates a paradigm for future investigation of the ADAM10 physiological role at the presynaptic terminal. ADAM10 is subjected to N-glycosylation and proteolysis $(28,29)$, which can modulate the interaction with its protein partners. The impact of these modifications on the reduced formation of the m-ADAM10/PCLO complex in HD remains unexplored. Additional studies are also needed to understand the contribution of presynaptic and postsynaptic ADAM10 to HD cortico-striatal dysfunction. Our previous findings indicate that hyperactive ADAM10 in the postsynaptic terminal causes excessive proteolysis of the synaptic cell adhesion protein N-CAD. Notably, postsynaptic N-CAD proteolysis has been proposed to influence SVs dynamics and release from the presynaptic terminal (30-33). Targeting ADAM10 activity selectively in the presynaptic or postsynaptic terminal will help to clarify the relevance of this metalloprotease in the two compartments and to identify the primary target for future ADAM10modulating therapies.

\section{Materials and Methods}

\section{Animal models, genotyping and ethical approval}

HD rodent models. Wild-type and $\mathrm{R} 6 / 2$ mice (B6CBAF1/J) were purchased from the Jackson Laboratory. Wildtype and zQ175 (C57BL/6J) mice were obtained from the CHDI Foundation (https://www.jax.org/strain/027410). Genotyping of R6/2 (B6CBAF1/J) and zQ175 (C57BL/6J) mouse colonies ( 150 CAG and 175 repeats, respectively) was performed by Polymerase Chain Reaction (PCR) of genomic DNA obtained from tail samples (Nucleo Spin Tissue, Macherey-Nagel, catalog 740952.250) at weaning and following sacrifice for verification. CAG repeats of R6/2 mice were sized by using the following PCR primers: forward: 5'- 


\section{ATGAAGGCCTTCGAGTCCCTCAAGTCCTTC-3'; reverse: 5'-GGCGGCTGAGGAAGCTGAGGA-3' .}

Cycling conditions were $90^{\circ} \mathrm{C}$ for 90 seconds, 35 cycles $\left(94^{\circ} \mathrm{C}\right.$ for 60 seconds, $65^{\circ} \mathrm{C}$ for 45 seconds, $72^{\circ} \mathrm{C}$ for 60 seconds), $72^{\circ} \mathrm{C}$ for 7 minutes. CAG repeats of $\mathrm{zQ} 175$ mice were sized by using the following PCR primers: forward: 5'-CATTCATTGCCTTGCTGCTAAG-3'; reverse: 5'-CTGAAACGACTTGAGCGACTC-3'. Cycling conditions were $94^{\circ} \mathrm{C}$ for 10 minutes, 30 cycles $\left(96^{\circ} \mathrm{C}\right.$ for 30 seconds, $57^{\circ} \mathrm{C}$ for 30 seconds, $72^{\circ} \mathrm{C}$ for 30 seconds), $72^{\circ} \mathrm{C}$ for 7 minutes.

Generation of R6/2-A10cKO mice. R6/2 mice (B6CBAF1/J) were generated by crossing R6/2 males with wildtype females (B6CBAF1/J). The A10cKO colony was maintained by crossing heterozygous floxed mice $\left(\right.$ Adam10 $\left.{ }^{F l o x /+}\right)(\mathrm{C} 57 \mathrm{BL} / 6 \mathrm{~J} \times 129 \mathrm{S6})$ with CaMKIIalpha-Cre recombinase transgenic mice $(\mathrm{C} 57 \mathrm{BL} / 6 \mathrm{~J} \times 129 \mathrm{~S} 6)$. Heterozygous Adam10 floxed mice $\left(\right.$ Adam10 $\left.{ }^{\text {Flox/+ }}\right)(\mathrm{C} 57 \mathrm{BL} / 6 \mathrm{~J} \times 129 \mathrm{~S} 6)$ and CaMKIIAlpha-Cre recombinase transgenic mice $(\mathrm{C} 57 \mathrm{BL} / 6 \mathrm{~J} \times 129 \mathrm{S6})$ were generated as described in (16). A10cKO exhibited a normal phenotype and a normal fertility. From the crossing between heterozygous A10cKO and R6/2 mice, we tested 4 genotypes of the F1, including wild-type, R6/2, R6/2-A10cKO, and A10cKO, which are on the same mixed genetic background. Mice were genotyped as described in (16).

Ethical approval. All protocols involving animals were carried out in accordance with institutional guidelines in compliance with Italian law (D. Lgs no. 2014/26, implementation of the 2010/63/UE, protocol 324/2015-PR). The Ethics Committee of the University of Milano approved studies in mice (Ethics Committee 09.10.13, Ethical Approval 74/13; Ethics Committee 18.12.13, Ethical Approval 74/14).

Immunoprecipitation, liquid chromatography with tandem mass spectrometry (LC-MS/MS) analysis and identification of endogenous ADAM10 interacting proteins from striata of wild-type and zQ175 mice Brain tissues from $n=4$ wild-type and $n=4$ homozygous 50-week-old $z Q 175$ mice were pestled in a $\mathrm{LN}_{2}$ pre-chill mortar to turn tissue samples to a fine powder. Mouse tissue homogenization was carried out by 20 strokes in a Teflon-glass homogenizer (clearance $0.25 \mathrm{~mm}, 700 \mathrm{rpm}$ ) in RIPA buffer (50 mM Tris $\mathrm{HCl} \mathrm{pH} 8,150 \mathrm{mM} \mathrm{NaCl}$, $0.1 \%$ sodium dodecyl sulfate (SDS), $1 \%$ NP40 with $1 \mathrm{mM}$ phenylmethylsulfonyl fluoride (PMSF) and $1 \times$ protease inhibitor cocktail (Roche Scientific, catalog 11836170001). Tissue lysates were then passed through 25 gauge needle attached on a $1 \mathrm{ml}$ syringe. After 20 minutes in ice, lysates were put 15 minutes on a rotating wheel at $4^{\circ} \mathrm{C}$. Lysates were then cleared by centrifugation for 30 minutes at $12,000 \mathrm{~g}$ and $4^{\circ} \mathrm{C}$. Resulting supernatants were collected and protein concentrations determined by Bradford method with Bio-Rad Protein 
Assay Dye Reagent Concentrate (Bio-Rad, catalog 5000006). Tissue lysates from $n=4$ mice/genotypes were pooled and $6 \mathrm{mg}$ of protein lysate for wild-type and zQ175 mice was pre-cleared using Dynabeads Protein G (Thermo Fisher Scientific, catalog 10004D) according to the manufacturer's instructions and then immunoprecipitated by using $24 \mu 1$ of rabbit polyclonal anti-ADAM10 provided by P. Saftig (University of Kiel). The elution from pre-clearing (control) and immunoprecipitated beads was carried out in Sample Buffer $2 \times(200$ $\mathrm{mM}$ Tris $\mathrm{HCl} \mathrm{pH}$ 6.8, 40\% glycerol, 8\% SDS, $200 \mathrm{mM}$ dithiothreitol (DTT), $0.01 \%$ bromophenol blue) at $99^{\circ} \mathrm{C}$ for 10 minutes. Eluted samples were fractionated by sodium dodecyl sulfate-polyacrylamide gel electrophoresis (SDS-PAGE), and stained with GelCode ${ }^{\mathrm{TM}}$ Blue Safe Protein Stain (Thermo Fisher Scientific, catalog 24592 ). Whole SDS-PAGE lanes were cut in $1 \mathrm{~mm}$ slices and the gel bands digested in situ with trypsin as previously reported in (34). Peptide mixtures were analysed by nano LC-MS/MS using LTQ Orbitrap XL mass spectrometer coupled to Proxeon nanoEasy II capillary HPLC (Thermo Fisher Scientific Inc., Waltham, MA). Peptides were fractionated onto a C18 reverse-phase capillary column ( $3 \mu \mathrm{m}$ biosphere, $7.5 \mu \mathrm{m}$ internal diameter, $100 \mathrm{~mm}$ length) working at $300 \mathrm{nl} / \mathrm{min}$ flow rate and adopting a linear gradient from $5 \%$ to $100 \%$ of eluent $\mathrm{B}(0.2 \%$ formic acid, 95\% acetonitrile LC-MS Grade) over 49 min. Mass spectrometric analyses were carried out in Data Depending Acquisition (DDA): from each MS scan, spanning from 400 to $1800 \mathrm{~m} / \mathrm{z}$, the five most abundant ions were selected and fragmented. Output data were further processed into mgf files suitable for protein identification procedure by Mascot licensed software (Matrix Science Boston, USA). Proteins were searched in NCBI database using Proteome Discoverer v1.4 platform (Thermo Fisher Scientific). Proteins identification was carried out by using $10 \mathrm{ppm}$ as peptides mass tolerance for MS and 0.6 Da for MS/MS search; Mus Musculus as taxonomy, carbamidomethyl (C) as fixed modification and Gln->pyro-Glu (N-term Q), Oxidation (M), Pyrocarbamidomethyl (N-term C) as variable modifications.

\section{Filtering out immunoprecipitation contaminants}

For the definition of the proteins that are non-specific contaminants of immunoprecipitation, we relied on the Contaminant Repository for Affinity Purification (CRAPome) 2.0 web tool (https://reprint-apms.org), querying the human experiment collection. To this end, starting from the list of Mouse Genome Informatics (MGI) symbols for ADAM10 interactors in wild-type and zQ175 mice, human homolog genes were retrieved by biomaRt R package (Ensembl version 98, (35). CRAPome was then interrogated using 'Workflow1' and 'H. sapiens - All' parameter. Contaminants were defined as proteins reported in at least $50 \%$ of the experiments. 


\section{Generation of ADAM10 interaction network}

A network visualizing ADAM10 interactors in wild-type and zQ175 striata was generated in Cytoscape software (version 3.7.2). Interactors are identified by their MGI gene symbol and are visualized as nodes connected to ADAM10 in wild-type and/or mutant conditions.

\section{Gene Ontology Enrichment Analysis of ADAM10 interactors}

ADAM10 interactors identified for wild-type and zQ175 mice (160 and 130 proteins respectively, including ADAM10) were subjected to gene ontology enrichment analysis. To define a universe of expressed proteins to be used as background reference in the enrichment calculation, the ones identified by the striatal proteomic analysis performed in (19) were considered. Starting from the article supplementary information i.e. Table S13, proteins with a Sufficient Values of 1 and with Uniprot ID referring to a single gene symbol were selected, resulting in a final list of 7197 proteins whose MGI symbol was used as universe. Gene ontology enrichment analysis was performed in R with TopGO package (http://bioconductor.org/packages/release/bioc/html/topGO.html) considering the Cellular Component domain of the ontology and using the Fisher statistics and the Weight01 algorithm. Terms were considered significant with a $\mathrm{P}$ Value $<0.01$ and an enrichment value of at least 2 . The top-three categories (ranked by P value) in both wild-type and zQ175 mice were: (I) glutamatergic synapse; (II) post-synaptic density; (III) pre-synaptic active-zone cytoplasmic component. Starting from the interactome generated from the proteomic data, the genes associated with 'glutamatergic synapse' category were highlighted in the network as blue nodes.

\section{Transcriptional dysregulation of ADAM10 interactors by HTT}

To further characterize the proteins identified as ADAM10 interactors in the wild-type and zQ175 striatum, we explored their transcriptional modulation in zQ175 mice by interrogating the data produced by (19). Results of differential gene expression analysis, performed on the striatum of 10-month old zQ20 and zQ175 mice, were interrogated, retrieving the FC and FDR for the interactors; information was not available for the following proteins: EPB41L3, MAP6, MTCO2, OPA, PC, RAB1A, RAB7A, SERPINB6, SKT, SEPT6, SEPT9, SYNGAP1, UPF0568, ZC2HC1A. For the other interactors, FDR and FC values were imported in Cytoscape and displayed as followed: (I) node color code according to FC: red for down-regulated genes, green for up-regulated 
genes; (II) node and label size according to - $\log 10 \mathrm{FDR}$. Genes for which the information is not available: grey color. Labels are shown only for nodes with FDR $<0.1$.

\section{Overlap between ADAM10 and HTT interactomes}

HTT interactors were retrieved from HdinHd website (https://hint.hdinhd.org/interactions/); mouse and human interactors were retained for the analysis. For human interactors, the corresponding mouse homolog genes were retrieved by biomaRt R package (Ensembl version 98, (35) and then added to the mouse interactors. After removal of duplicates, a list of 1625 HTT interactors (as MGI symbols) was obtained and used for the overlap with ADAM10 interactors in wild-type and zQ175 mice. Results of the overlap were represented by a Venn diagram, produced by R package VennDiagram (36); shared interactors were also visualized in cytoscape network, by adding HTT as a node with edges connected to shared interactors.

Gene ontology enrichment analysis was performed on the proteins shared between HTT and ADAM10 (both wild-type and zQ175, for a total of 105) using the same approach and thresholds already detailed for gene ontology enrichment analysis of ADAM10 interactors.

\section{Preparation of synaptosomes, pre- and postsynaptic fractions}

Synaptosomes from cortical tissue of wild-type, R6/2, R6/2-A10cKO shown in Fig. 3F and in Supplementary Material, Fig. S4 were prepared by using Syn-PER Reagent (Thermo Scientific, catalog 87793) according to the manufacturer's instructions. For the preparation of synaptosomes, pre- and postsynaptic fractions from cortical tissue of wild-type and R6/2 mice shown in Fig. 3A-C, tissue homogenization was carried out by 10 strokes in a Teflon-glass homogenizer (clearance $0.25 \mathrm{~mm}, 700 \mathrm{rpm}$ ) in $4 \mathrm{ml} / \mathrm{g}$ wet weight cold $0.32 \mathrm{M}$ sucrose solution containing $1 \mathrm{mM} \mathrm{NaHCO} 3,1 \mathrm{mM} \mathrm{MgCl}_{2}, 0.5 \mathrm{mM} \mathrm{CaCl}_{2}, 1 \mathrm{mM}$ PMSF, and $1 \times$ protease inhibitors (Thermo Fisher Scientific, catalog 1861281) (Buffer A). The homogenized tissue was centrifuged at $470 \mathrm{~g}$ for 2 minutes at $4^{\circ} \mathrm{C}$. The resulting supernatant (S1) was centrifuged at $10,000 \mathrm{~g}$ for 10 minutes at $4^{\circ} \mathrm{C}$, and the pellet $(\mathrm{P} 2)$ was resuspended in $0.32 \mathrm{M}$ sucrose solution and layered on $0.8 \mathrm{M}$ sucrose solution followed by centrifugation at 9,100 $g$ for 15 minutes at $4^{\circ} \mathrm{C}$ in swinging bucket rotor. Synaptosomes in the 0.8 sucrose fraction were resuspended in $20 \mathrm{mM}$ HEPES pH 7, 2\% Triton X-100, $150 \mathrm{mM} \mathrm{KCl,} 1 \mathrm{mM}$ PMSF, and 1× protease inhibitors (Buffer B) and centrifuged at 20,800 $g$ for 45 minutes. The resulting supernatant (S3) represents the presynaptic fraction, while the pellet (P3) was resuspended in $1 \%$ Triton-X 100, $75 \mathrm{mM} \mathrm{KCl,} 1 \mathrm{mM}$ PMSF, and $1 \times$ protease inhibitors 
(Buffer C) and centrifuged at 20,800 $\mathrm{g}$ for 30 minutes at $4^{\circ} \mathrm{C}$. The obtained pellet (P4), which represents the postsynaptic fraction, was washed with $20 \mathrm{mM}$ HEPES $\mathrm{pH} 7$ and resuspended in 1× SDS-PAGE buffer (100 mM Tris $\mathrm{HCl}$ pH 6.8, 20\% glycerol, 4\% SDS, $100 \mathrm{mM}$ DTT, 0.01\% bromophenol blue). Proteins in the presynaptic fractions were precipitated with trichloroacetic acid (TCA) stock to 4 volumes of protein sample and incubated 10 minutes at $4{ }^{\circ} \mathrm{C}$. Samples were centrifuged at $14,000 \mathrm{rpm}$ for 5 minutes. Pellets were washed 2 times with cold acetone, centrifuged at $14,000 \mathrm{rpm}$ for $5 \mathrm{~min}$ and dried in $95^{\circ} \mathrm{C}$ heat block for 5-10 minutes to drive off acetone, and resuspended in $1 \times$ SDS-PAGE buffer. Densitometric quantification of protein loads stained with InstantBlue ${ }^{\circledR}$ Coomassie Protein Stain (Abcam, catalog ab119211) allowed to load equal amounts of protein lysates on $10 \%$ SDS-PAGE gels for Western Blot analyses.

\section{Co-IP of the ADAM10/PCLO complex}

Co-IP was performed on total protein lysates and on synaptosomes prepared from cortical tissues of wild-type and R6/2 mice at 12 weeks of age.

Co-IP of ADAM10/PCLO complex in total protein lysates. Tissue homogenization was carried out in RIPA buffer (50 mM Tris $\mathrm{HCl} \mathrm{pH} 8,150 \mathrm{mM} \mathrm{NaCl}, 0.1 \%$ SDS, $1 \%$ NP40 with $1 \mathrm{mM}$ PMSF and $1 \times$ protease inhibitor cocktail (Roche Scientific, catalog 11836170001). Tissue lysates were then passed through 25gauge needle attached on a 1 $\mathrm{ml}$ syringe. After 20 minutes in ice lysates were put 15 minutes on a rotating wheel at $4^{\circ} \mathrm{C}$. Lysates were then cleared by centrifugation for 30 minutes at $12,000 \mathrm{~g}$ and $4{ }^{\circ} \mathrm{C}$. Resulting supernatants were collected and protein concentrations determined by Bradford method with Bio-Rad Protein Assay Dye Reagent Concentrate (Bio-Rad, catalog 5000006). $1 \mathrm{mg}$ protein lysate was pre-cleared using Dynabeads Protein G (Thermo Fisher Scientific, catalog 10004D) according to the manufacturer's instructions and then incubated with $50 \mu 1$ of rabbit polyclonal anti-PCLO antibody (Synaptic Systems, catalog 142002) over night at $4^{\circ} \mathrm{C}$ on a rotation wheel and then loaded onto Dynabeads (Thermo Fisher Scientific, catalog 10004D) according to the manufactured instruction, and incubated for 3 hours at $4^{\circ} \mathrm{C}$ on a rotation wheel. After washing, the beads were eluted in Sample Buffer $2 \times(200$ mM Tris $\mathrm{HCl} \mathrm{pH} \mathrm{6.8,} \mathrm{40 \%} \mathrm{glycerol,} \mathrm{8 \%} \mathrm{SDS,} 200 \mathrm{mM}$ DTT, $0.01 \%$ bromophenol blue) at $99^{\circ} \mathrm{C}$ for 10 minutes. For Western blot, Co-IP samples were denatured at $99^{\circ} \mathrm{C}$ and loaded on a $6 \%$ and $10 \%$ SDS-PAGE gels. Input samples (5\% of the amount of the corresponding lysate used for IP) were loaded as controls.

Co-IP of ADAM10/PCLO in synaptosomes. The Syn-PER Reagent (Thermo Scientific, catalog 87793) was used for synaptosomes preparation from cortical tissue of wild-type, R6/2 and R6/2-A10cKO at 12 weeks of age. A 
pool of $n=3$ mice/genotype was used and $1 \mathrm{mg}$ synaptosomes were pre-cleared using SureBeads ${ }^{\mathrm{TM}}$ Protein $\mathrm{G}$ Magnetic Beads (Bio-Rad, catalog 161-4023) according to the manufacturer's instructions. Pre-cleared samples were incubated with $10 \mu 1$ of rabbit polyclonal anti-PCLO antibody (Synaptic Systems, catalog 142002) over night at $4{ }^{\circ} \mathrm{C}$ on a rotation wheel and then loaded onto SureBeads ${ }^{\mathrm{TM}}$ Protein G Magnetic Beads according to the manufactured instruction. After washing, samples were eluted in $60 \mu 1 /$ sample of Sample Buffer $2 \times(200 \mathrm{mM}$ Tris $\mathrm{HCl} \mathrm{pH} \mathrm{6.8,} \mathrm{40 \%} \mathrm{glycerol,} 8 \%$ SDS, $200 \mathrm{mM}$ DTT, $0.01 \%$ bromophenol blue) at $65^{\circ} \mathrm{C}$ for 20 minutes. IP samples and inputs ( $5 \%$ of the amount of the corresponding lysate used for IP) were then loaded on 3-8\% Criterion ${ }^{\mathrm{TM}} \mathrm{XT}^{\prime}$ Tris-Acetate Protein Gel (Bio-Rad, catalog 3450129).

\section{Western Blot}

Separated proteins were transferred onto a nitrocellulose membrane (Bio-Rad Turbo Nitrocellulose, catalog 1704158) by means of the Trans-blot Turbo Transfer System (Bio-Rad) (High Molecular Weight protocol: 2.5 A constant; up to $25 \mathrm{~V}$; 10 minutes), blocked with 5\% nonfat milk (Bio-Rad, catalog 1706404) in TBS and 0.1\% Tween 20 (TBST), and incubated with rabbit polyclonal anti-ADAM10 provided by P. Saftig (1:1000 in TBST), with mouse monoclonal anti-PSD95 antibody (1:2000 in TBST; Thermo Fisher Scientific, catalog 6G6-1C9), with mouse monoclonal anti-SYP antibody (1:500 in 5\% nonfat milk; Abcam, catalog ab8049), with rabbit polyclonal anti-PCLO antibody (1:2000 in 5\% nonfat milk; Synaptic System, catalog 142002), with mouse monoclonal anti-RNA polymerase II CTD antibody (1:1000 in TBST; Abcam, catalog ab5408), with mouse monoclonal anti- $\beta$ III-Tubulin (1:1000in TBST; Promega, catalog G7121), and mouse monoclonal anti $\alpha$-Tubulin (1:5000 in TBST; MilliporeSigma, catalog T9026) at $4^{\circ} \mathrm{C}$ overnight. After washing, filters were incubated for 1 hour at room temperature with a peroxidase conjugate secondary antibody (1:3000 in 5\% nonfat milk; Bio-Rad, goat anti-rabbit HRP, catalog 1706515, goat anti-mouse HRP, catalog 1706516) and then washed 3 times with TBST. Co-IP membranes were incubated with TidyBlot (1.500 in TBS-T, Bio-Rad, catalog STAR209P). The Clarity Western ECL Substrate (Bio-Rad, catalog 1705061) was used to visualize immunoreactive bands. Blot visualization was performed by using the ChemiDoc MP Imaging System from Bio-Rad. Densitometric analyses were performed by using Image Lab version 6.0.1.

\section{Transmission Electron Microscopy (TEM)}


Wild-type, R6/2, R6/2-A10cKo and A10cKO mice at 13 weeks of age were anesthetized with $10 \mathrm{mg} / \mathrm{ml}$ avertin and transcardially perfused using 2.5\% glutaraldehyde (Electron Microscopy Sciences, catalog 16220), and 2\% paraformaldehyde (Electron Microscopy Sciences, catalog 19200) as fixatives, both in sodium cacodylate buffer $0.15 \mathrm{M}$ (pH 7.4) (Electron Microscopy Sciences, catalog 12300). Brains were removed and post-fixed for an additional 24 hours at $4{ }^{\circ} \mathrm{C}$. Coronal brain sections (100 $\mu \mathrm{m}$ thick) were cut on a Leica VT1000S Vibratome. Sections were collected in $0.1 \mathrm{M}$ sodium cacodylate buffer, and the cortex was manually dissected for staining and embedding. Tissues were washed with $0.1 \mathrm{M}$ cold sodium cacodylate buffer and then postfixed in a reduced osmium solution (i.e., 1.5\% potassium ferrocyanide, Electron Microscopy Sciences, catalog 20150) with $2 \%$ osmium tetroxide (Electron Microscopy Sciences, catalog 19170) in $0.15 \mathrm{M}$ cacodylate buffer for 1 hour on ice. After the first heavy metal incubation, the tissues were washed with $\mathrm{ddH}_{2} \mathrm{O}$ at room temperature and then placed in the $0.22 \mu \mathrm{m}$ Millipore-filtered 1\% thiocarbohydrazide (TCH) (Electron Microscopy Sciences, catalog 21900) in $\mathrm{ddH}_{2} \mathrm{O}$ solution for 20 minutes at room temperature. Tissues were then rinsed again in $\mathrm{ddH}_{2} \mathrm{O}$ and incubated in $2 \%$ osmium tetroxide in $\mathrm{ddH}_{2} \mathrm{O}$ for 30 minutes at room temperature. After several washings at room temperature in $\mathrm{ddH}_{2} \mathrm{O}$, they were then placed in $1 \%$ uranyl acetate (aqueous) and left overnight at $4^{\circ} \mathrm{C}$.Samples were washed once again and then immersed en bloc in Walton's lead aspartate solution ( $0.066 \mathrm{gr}$ lead nitrate, Electron Microscopy Sciences, catalog 17900) dissolved in $10 \mathrm{ml}$ of $0.003 \mathrm{M}$ aspartic acid solution, $\mathrm{pH} 5.5$ ) at $60^{\circ} \mathrm{C}$ for 30 minutes. The tissues were washed and then dehydrated stepwise through an ethanol series and finally placed in anhydrous ice-cold acetone for 10 minutes. Infiltration was performed with an acetone (Sigma-Aldrich, catalog 179124)/Durcupan ACM (Electron Microscopy Sciences, catalog 14040) mixture with 3:1 volume ratio for 2 hours, then 1:1 overnight. The tissues were left for 2 hours in pure resin and then embedded in Durcupan ACM resin and placed in a $60^{\circ} \mathrm{C}$ oven for 48 hours for polymerization. For TEM imaging, ultrathin sections $70 \mathrm{~nm}$ thick were prepared by a Leica UC7 ultramicrotome and placed on TEM copper grids (Electron Microscopy Sciences, catalog EMS300-Cu), which were then observed using a Tecnai Spirit transmission electron microscope (FEI, Eindheven, Netherlands) working at an acceleration voltage of $120 \mathrm{kV}$, equipped with a lanthanum hexaboride thermionic source, a twin objective lens and a bottom-mount Gatan Orius CCD camera (Gatan, Pleasanton, US). Quantitative measurements were performed by ImageJ, version 1.47 (NIH). Selection of the synapse and the analyses were performed by a blinded independent investigator and in a genotype blinded manner. The following parameters were measured: SVs number per area unit $\left(\mu \mathrm{m}^{2}\right)$, number of docked/reserve/resting vesicles per synaptic area $\left(\mathrm{N}^{\circ} \mathrm{SVs} / \mu \mathrm{m}^{2}\right)$, synaptic area $\left(\mu \mathrm{m}^{2}\right)$, AZ length $(\mu \mathrm{m})$, SVs diameter and PSD length $(\mathrm{nm})$. For SVs 
distribution single vesicle has been manually annotated and the distance to the active zone is expressed in $\mathrm{nm}$. Docked vesicles were defined as vesicles within $50 \mathrm{~nm}$ of the active zone, reserve as vesicles between $50 \mathrm{~nm}$ and $300 \mathrm{~nm}$ from the active zone and resting as vesicles beyond $300 \mathrm{~nm}$ of the active zone. We analyzed $\mathrm{n}=3$ mice/genotypes and $\mathrm{n}=60$ synapse/genotypes.

\section{Statistics}

All measurements are presented as mean \pm SEM. Prism 7.0c (GraphPad Software) was used to perform all statistical analyses. The D'Agostino-Pearson or Shapiro-Wilk normality test were used to determine in Prism whether data were normally distributed or not. For normally distributed values, statistical significance was determined by the unpaired Student's $t$ test when 2 groups were compared. When more than 2 groups were tested the one-way ANOVA with Tukey's multiple comparisons test was used. Differences were considered statistically significant at $P<0.05$.

\section{Acknowledgements}

We acknowledge the CHDI Foundation for providing zQ175 mice.

Conflict of interest statement. None declared.

\section{Funding}

This study was supported by Telethon (GGP13053) to CZ, Ministero dell'Istruzione, dell'Università e della Ricerca Scientifica, Programmi di Ricerca Scientifica di Rilevante Interesse Nazionale (20128XWKTX) to CZ and MM, ModelPolyQ (JPCOFUND-643417) and CircProt (JPCOFUND-643417) to EC, Deutsche Forschungsgemeinschaft (DFG-SFB877-A3) to PS, and KAUST baseline funding to AF. 


\section{References}

1 Saftig, P. and Lichtenthaler, S.F. (2015) The alpha secretase ADAM10: A metalloprotease with multiple functions in the brain. Prog. Neurobiol., 135, 1-20.

2 Marcello, E., Gardoni, F., Mauceri, D., Romorini, S., Jeromin, A., Epis, R., Borroni, B., Cattabeni, F., Sala, C., Padovani, A. et al. (2007) Synapse-associated protein-97 mediates $\alpha$-secretase ADAM10 trafficking and promotes its activity. J. Neurosci., 27, 1682-1691.

3 Marcello, E., Saraceno, C., Musardo, S., Vara, H., de La Fuente, A.G., Pelucchi, S., Di Marino, D., Borroni, B., Tramontano, A., Pérez-Otaño, I. et al. (2013) Endocytosis of synaptic ADAM10 in neuronal plasticity and Alzheimer's disease. J. Clin. Invest., 123, 2523-2538.

4 Kuhn, P.-H., Colombo, A.V., Schusser, B., Dreymueller, D., Wetzel, S., Schepers, U., Herber, J., Ludwig, A., Kremmer, E. and Montag, D. (2016) Systematic substrate identification indicates a central role for the metalloprotease ADAM10 in axon targeting and synapse function. Elife, 5, 2050-2084.

5 Endres, K. and Deller, T. (2017) Regulation of alpha-secretase ADAM10 in vitro and in vivo: genetic, epigenetic, and protein-based mechanisms. Front. Mol. Neurosci., 10, 56.

6 Malinverno, M., Carta, M., Epis, R., Marcello, E., Verpelli, C., Cattabeni, F., Sala, C., Mulle, C., Di Luca, M. and Gardoni, F. (2010) Synaptic localization and activity of ADAM10 regulate excitatory synapses through N-cadherin cleavage. J. Neurosci., 30, 16343-16355.

7 Restituito, S., Khatri, L., Ninan, I., Mathews, P.M., Liu, X., Weinberg, R.J. and Ziff, E.B. (2011) Synaptic autoregulation by metalloproteases and $\gamma$-secretase. J. Neurosci., 31, 12083-12093.

8 Andreyeva, A., Nieweg, K., Horstmann, K., Klapper, S., Müller-Schiffmann, A., Korth, C. and Gottmann, K. (2012) C-terminal fragment of N-cadherin accelerates synapse destabilization by amyloid- $\beta$. Brain, 135, 2140-2154.

9 MacDonald, M.E., Ambrose, C.M., Duyao, M.P., Myers, R.H., Lin, C., Srinidhi, L., Barnes, G., Taylor, S.A., James, M. and Groot, N. (1993) A novel gene containing a trinucleotide repeat that is expanded and unstable on Huntington's disease chromosomes. Cell, 72, 971-983.

10 Zuccato, C., Valenza, M. and Cattaneo, E. (2010) Molecular mechanisms and potential therapeutical targets in Huntington's disease, Physiol. Rev., 90, 905-981.

11 Saudou, F. and Humbert, S. (2016) The biology of huntingtin. Neuron, 89, 910-926.

12 Veldman, M.B. and Yang, X.W. (2018) Molecular insights into cortico-striatal miscommunications in Huntington's disease. Curr. Opin. Neurobiol., 48, 79-89.

13 Sardo, V.L., Zuccato, C., Gaudenzi, G., Vitali, B., Ramos, C., Tartari, M., Myre, M.A., Walker, J.A., Pistocchi, A., Conti, L. et al. (2012) An evolutionary recent neuroepithelial cell adhesion function of huntingtinimplicates ADAM10-Ncadherin. Nat. Neurosci., 15, 713-721.

14 Vezzoli, E., Caron, I., Talpo, F., Besusso, D., Conforti, P., Battaglia, E., Sogne, E., Falqui, A., Petricca, L., Verani, M. et al. (2019) Inhibiting pathologically active ADAM10 rescues synaptic and cognitive decline in Huntington's disease. J. Clin. Invest., 129, 2390-2403.

15 Menalled, L.B., Kudwa, A.E., Miller, S., Fitzpatrick, J., Watson-Johnson, J., Keating, N., Ruiz, M., Mushlin, R., Alosio, W., McConnell, K. et al. (2012) Comprehensive behavioral and molecular characterization of a new knock-in mouse model of Huntington's disease: zQ175. PloS One, 7, e49838. 
16 Prox, J., Bernreuther, C., Altmeppen, H., Grendel, J., Glatzel, M., D'Hooge, R., Stroobants, S., Ahmed, T., Balschun, D., Willem, M. et al. (2013) Postnatal disruption of the disintegrin/metalloproteinase ADAM10 in brain causes epileptic seizures, learning deficits, altered spine morphology, and defective synaptic functions. J. Neurosci., 33, 12915-12928.

17 Seipold, L., Altmeppen, H., Koudelka, T., Tholey, A., Kasparek, P., Sedlacek, R., Schweizer, M., Bär, J., Mikhaylova, M., Glatzel, M. et al. (2018) In vivo regulation of the A disintegrin and metalloproteinase 10 (ADAM10) by the tetraspanin 15. Cell. Mol. Life Sci., 75, 3251-3267.

18 Mellacheruvu, D., Wright, Z., Couzens, A.L., Lambert, J.-P., St-Denis, N.A., Li, T., Miteva, Y.V., Hauri, S., Sardiu, M.E., Low, T.Y. et al. (2013) The CRAPome: a contaminant repository for affinity purification-mass spectrometry data. Nat. Methods, 10, 730-736.

19 Langfelder, P., Cantle, J.P., Chatzopoulou, D., Wang, N., Gao, F., Al-Ramahi, I., Lu, X.-H., Ramos, E.M., El-Zein, K. and Zhao, Y. (2016) Integrated genomics and proteomics define huntingtin CAG lengthdependent networks in mice. Nat. Neurosci., 19, 623.

20 Emperador-Melero, J. and Kaeser, P.S. (2020) Assembly of the presynaptic active zone. Curr. Opin. Neurobiol., 63, 95-103.

21 Mangiarini, L., Sathasivam, K., Seller, M., Cozens, B., Harper, A., Hetherington, C., Lawton, M., Trottier, Y., Lehrach, H., Davies, S.W. et al. (1996) Exon 1 of the HD gene with an expanded CAG repeat is sufficient to cause a progressive neurological phenotype in transgenic mice. Cell, 87, 493-506.

22 Cepeda, C., Wu, N., André, V.M., Cummings, D.M. and Levine, M.S. (2007) The corticostriatal pathway in Huntington's disease. Prog. Neurobiol., 81, 253-271.

23 Murmu, R.P., Li, W., Szepesi, Z. and Li, J.-Y. (2015) Altered sensory experience exacerbates stable dendritic spine and synapse loss in a mouse model of Huntington's disease. J. Neurosci., 35, 287-298.

24 Gundelfinger, E.D., Reissner, C. and Garner, C.C. (2016) Role of bassoon and piccolo in assembly and molecular organization of the active zone. Front. Synaptic Neurosci., 7, 19.

25 Ackermann, F., Schink, K.Q., Bruns, C., Izsvák, Z., Hamra, F.K., Rosenmund, C. and Garner, C.C. (2019) Critical role for Piccolo in synaptic vesicle retrieval. Elife, 8, e46629.

26 Leal-Ortiz, S., Waites, C.L., Terry-Lorenzo, R., Zamorano, P., Gundelfinger, E.D. and Garner, C.C. (2008) Piccolo modulation of Synapsin1a dynamics regulates synaptic vesicle exocytosis. J. Cell Biol., 181, 831-846.

27 Parthier, D., Kuner, T. and Körber, C. (2018) The presynaptic scaffolding protein Piccolo organizes the readily releasable pool at the calyx of Held. J. Physiol., 596, 1485-1499.

28 Escrevente, C., Morais, V.A., Keller, S., Soares, C.M., Altevogt, P. and Costa, J. (2008) Functional role of $\mathrm{N}$-glycosylation from ADAM10 in processing, localization and activity of the enzyme. Biochim Biophys Acta, 1780, 905-913.

29 Tousseyn, T., Thathiah, A., Jorissen, E., Raemaekers, T., Konietzko, U., Reiss, K., Maes, E., Snellinx, A., Serneels, L., Nyabi, O. et al. (2009) ADAM10, the rate-limiting protease of regulated intramembrane proteolysis of Notch and other proteins, is processed by ADAMS-9, ADAMS-15, and the $\gamma$ secretase. J Biol Chem, 284, 11738-11747.

30 Bozdagi, O., Valcin, M., Poskanzer, K., Tanaka, H. and Benson, D.L. (2004) Temporally distinct demands for classic cadherins in synapse formation and maturation. Mol. Cell. Neurosci., 27, 509-521. 
31 Jüngling, K., Eulenburg, V., Moore, R., Kemler, R., Lessmann, V. and Gottmann, K. (2006) Ncadherin transsynaptically regulates short-term plasticity at glutamatergic synapses in embryonic stem cellderived neurons. J. Neurosci., 26, 6968-6978.

32 Vitureira, N., Letellier, M., White, I.J. and Goda, Y. (2012) Differential control of presynaptic efficacy by postsynaptic N-cadherin and $\beta$-catenin. Nat. Neurosci., 15, 81.

33 Gottmann, K. (2008) Transsynaptic modulation of the synaptic vesicle cycle by cell-adhesion molecules. J. Neurosci. Res., 86, 223-232.

34 Venuto, S., Monteonofrio, L., Cozzolino, F., Monti, M., Appolloni, I., Mazza, T., Canetti, D., Giambra, V., Panelli, P., Fusco, C. et al. (2020) TRIM8 interacts with KIF11 and KIFC1 and controls bipolar spindle formation and chromosomal stability. J. Cancer Letters, 473, 98-106.

35 Durinck, S., Spellman, P.T., Birney, E. and Huber, W. (2009) Mapping identifiers for the integration of genomic datasets with the R/Bioconductor package biomaRt. Nat. Protoc., 4, 1184-1191.

36 Chen, H. and Boutros, P.C. (2011) VennDiagram: a package for the generation of highlycustomizable Venn and Euler diagrams in R. BMC Bioinformatics, 12, 1-7.

\section{Legends to Figures}




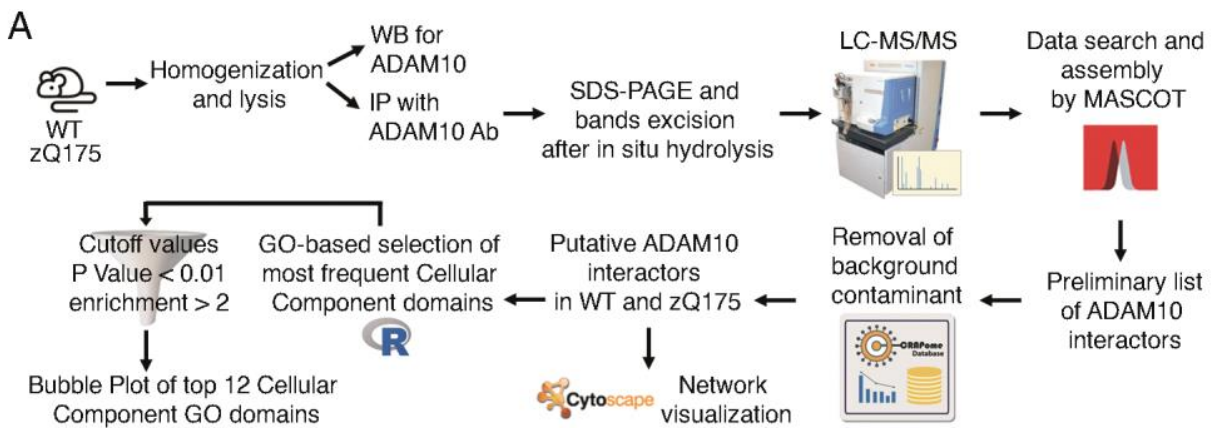

$\mathrm{B}$

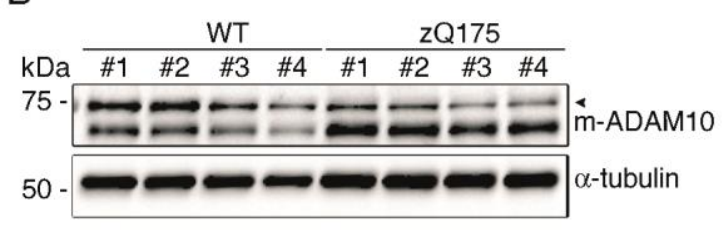

C

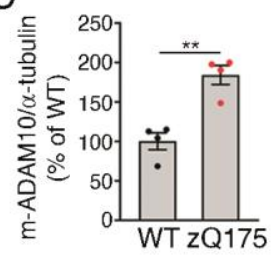

$\mathrm{D}$
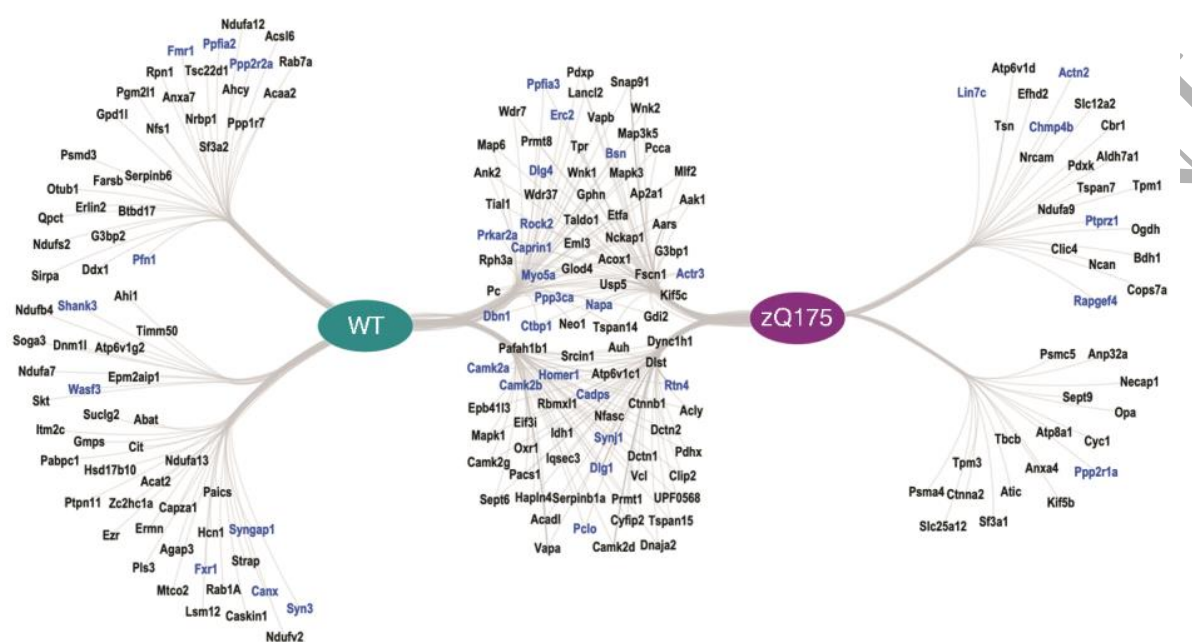

E

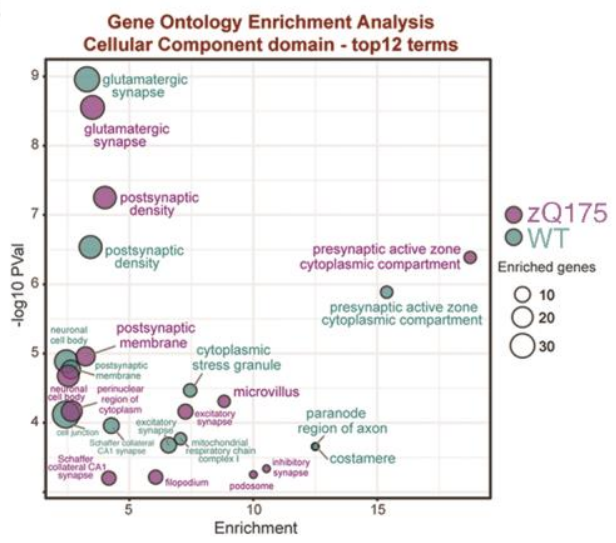

Cozzolino et al., Figure 1

Figure 1. Endogenous ADAM10 interaction in the striatum of HD mice.

(A) Workflow for the analysis of the ADAM10 interactome in the striatum of 50-week-old wild-type and homozygous zQ175 knock-in mice. 
(B,C) Representative Western blot and quantification of the level of m-ADAM10 in the striatum of wild-type $(n=4)$ and zQ175 striatum $(n=4)$ are shown. ${ }^{*} \mathrm{P}<0.05$ unpaired t-test. $\alpha$-tubulin was the loading control. The arrowhead indicates a non-specific band.

(D) Cytoscape network representing putative ADAM10 interactors, identified by their MGI gene symbols. Nodes (proteins) identified selectively in wild-type (ADAM10WT) and zQ175 (ADAM10MUT), and interactors identified in both the wild-type and zQ175 striatum are shown. Genes associated by Gene Ontology enrichment analysis with 'Glutamatergic synapse' are highlighted in blue.

(E) Gene Ontology enrichment analysis of the ADAM10 interactome in wild-type (green) or zQ175 (purple). The plot depicts the top-12 terms for each condition (ranked by their P value); the $\mathrm{x}$-axis reports the enrichment and $\mathrm{y}$ axis the significance (as $-\log 10 \mathrm{P}$ values), whereas the size of each dot is proportional to the number of genes assigned to the category. 
A

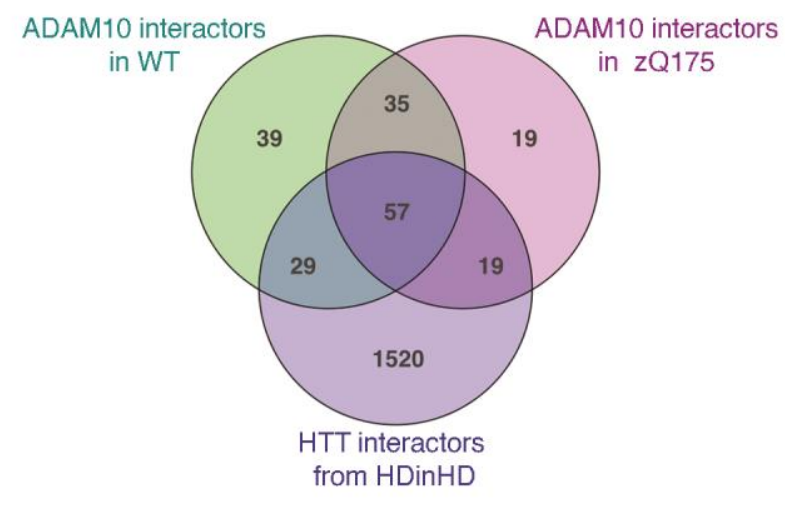

B

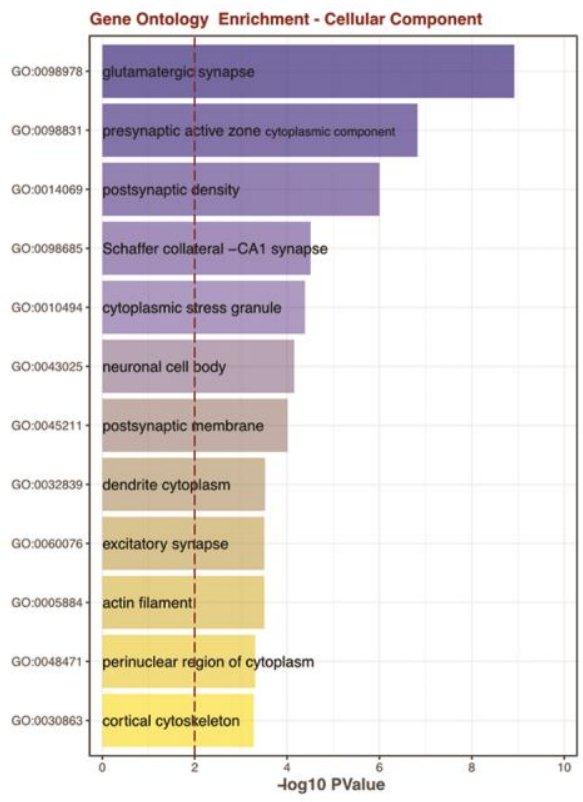

C

\begin{tabular}{|c|c|c|c|c|c|}
\hline Ppp1r7 & Suclg2 & Paics & Nis1 & Ndufa7 & Erlin2 \\
\hline Gmps & Soga3 & Zc2hc1a & Ndufb4 & Fxr1 & Wasf3 \\
\hline Nrbp1 & Skt & Acat2 & Serpinb6 6 & Abat & Gpd11 \\
\hline Tsc22d1 & Lsm12 & Pgm211 & Anxa7 & Shank3 & Syngap1 \\
\hline Mtco2 & Caskin1 & Syn3 & Pfn1 & Ndufa12 & Ppfia2 \\
\hline Rab1A & Pls3 & Epm2aip1 & $\mathrm{Sf3a2}$ & Rab7a & Ptpn11 \\
\hline $1 \mathrm{tm} 2 \mathrm{c}$ & Agap3 & Ermn & & & \\
\hline
\end{tabular}

retrieved from HDinHD). Fifty-seven interactors are shared among the three conditions; 105 are shared between HTT and ADAM10 when considering both wild-type and zQ175. 
(B) Gene Ontology enrichment analyses of putative interactors shared by HTT and ADAM10 in wild-type and zQ175 (105 interactors): the top-12 categories for the Cellular Component domain are shown, ranked by P-value.

(C) The identity of interactors shared between ADAM10 and HTT or specific for ADAM10 visualized by a

Cytoscape network. Interactors are identified by their MGI gene symbols. Blue: interactors shared between wildtype and zQ175; green: selective interactors for wild-type; pink: selective interactors for zQ175. Presynaptic proteins (identified by manual curation) that are putative ADAM10 and HTT interactors are indicated in red: BSN, bassoon; ERC2, ERC protein 2; CTNNB1, catenin beta 1; PCLO, piccolo; RPH3A, Rabphilin 3A; CTBP1, C-terminal binding protein 1; PPFIA3, liprin-alpha-3. 
A

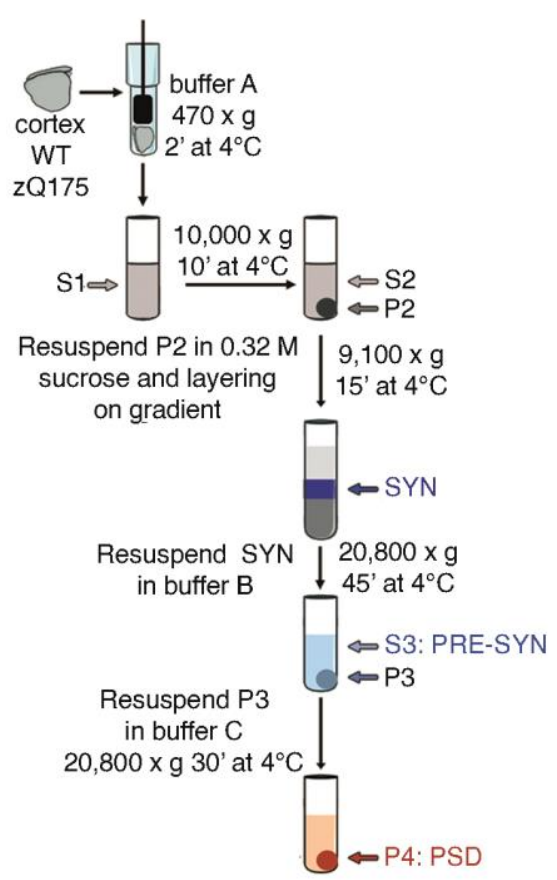

$\mathrm{D}$

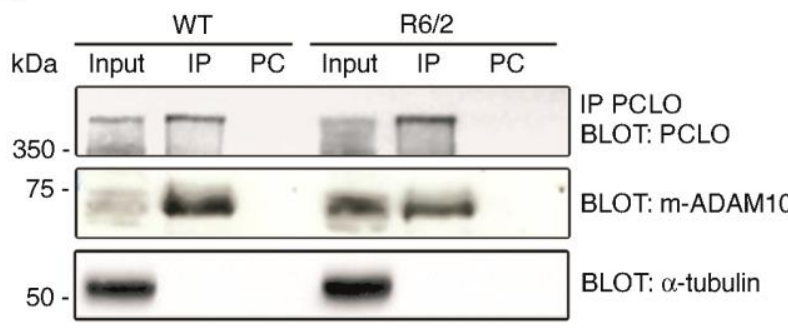

$\mathrm{F}$

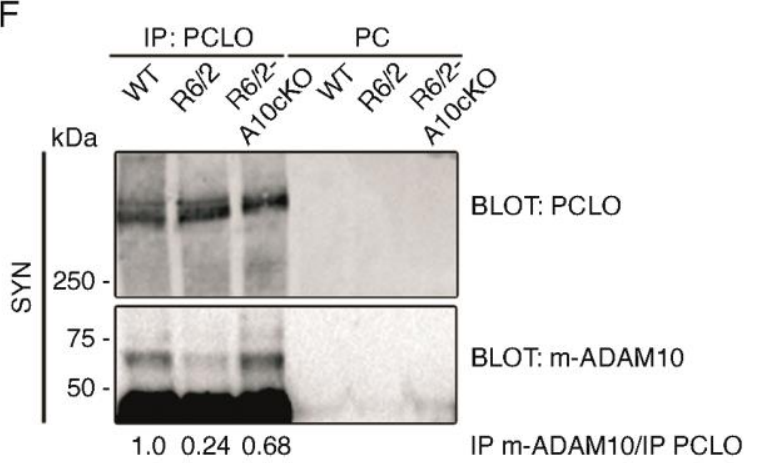

B

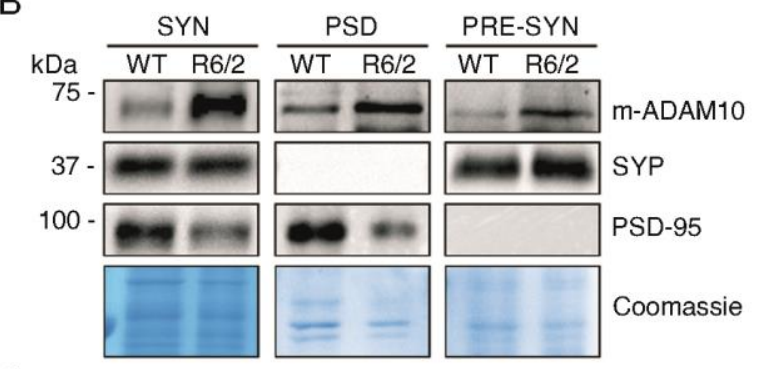

C

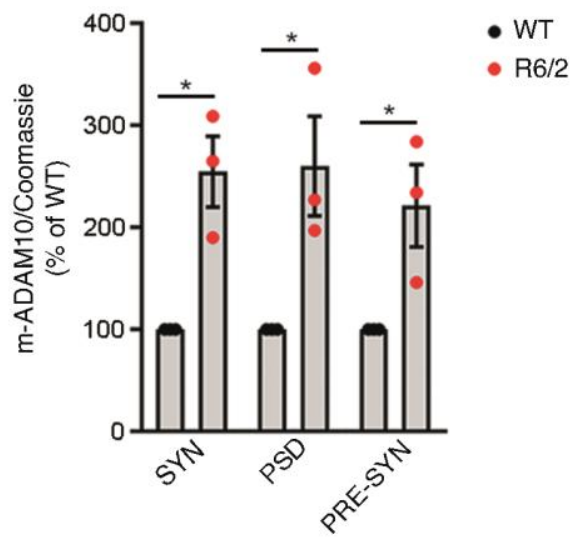

E
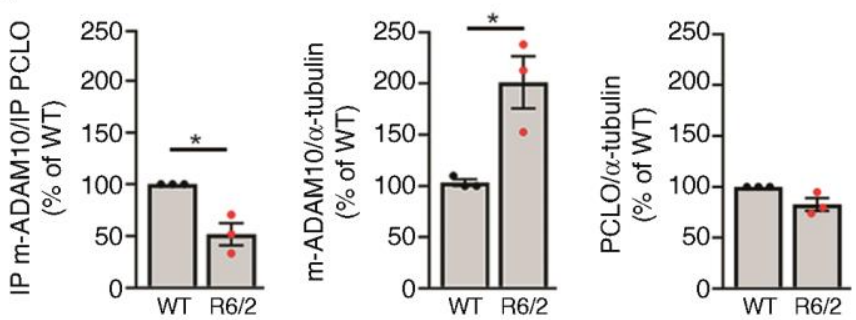

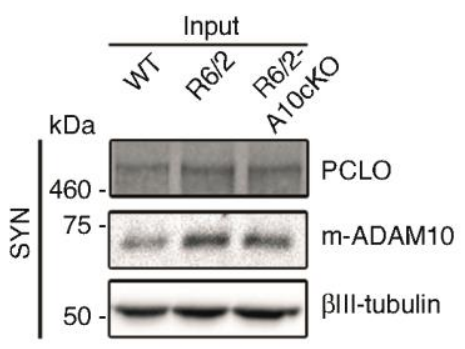

Cozzolino et al., Figure 3

Figure 3. ADAM10 at the presynaptic terminal and analysis of the ADAM10/PCLO complex in HD mice.

(A) Workflow of subcellular fractionation to enrich for synaptosomes (SYN), presynaptic (PRE-SYN) and postsynaptic fraction preparations (PSD).

(B) Representative Western blot for m-ADAM10 on SYN, PSD and PRE-SYN from a pool of three cortices from 12-week-old wild-type and R6/2 mice. Synaptophysin (SYP), PRE-SYN marker. PSD95, PSD marker. Coomassie staining was the loading control. 
(C) Quantification of data in B. Data are represented as mean \pm SEM of three biological replicates in which a pool of $\mathrm{n}=3$ mice/genotype was tested. $* \mathrm{P}<0.05$, unpaired t-test.

(D) Representative Co-IP experiment for the ADAM10/PCLO complex in cortical protein lysates prepared from a pool of two 12-week-old wild-type and R6/2 mice. PC, pre-clearing.

(E) Quantification of data in D. IP ADAM10/IP PCLO ratio is shown as well as level of m-ADAM10 and PCLO in the input samples. $\alpha$-tubulin was the loading control for the input samples. Data are represented as mean \pm SEM of $\mathrm{n}=3$ biological replicates in which a pool of $\mathrm{n}=2$ mice/genotype was tested. $* \mathrm{P}<0.05$, unpaired $\mathrm{t}$-test. (F) CaMKII $\alpha$-Cre:Adam10 ${ }^{\text {Flox/+ }}$ (A10cKO) mice were crossed with R6/2 mice to obtain R6/2:CaMKII $\alpha$ Cre:Adam10 ${ }^{\text {Flox/+ }}$ (R6/2-A10cKO). Co-IP experiments for the ADAM10/PCLO complex on cortical synaptosomes from a pool of three 12-week-old wild-type, R6/2, or R6/2-A10cKO mice. $\beta$ III-Tubulin was the loading control for the input samples. IP ADAM10/IP PCLO ratio is shown at the bottom of the IP panel. A representative image from three co-IP replicates is shown. See Supplementary Material, Fig. S5 for data analysis of the co-IP technical replicates. 


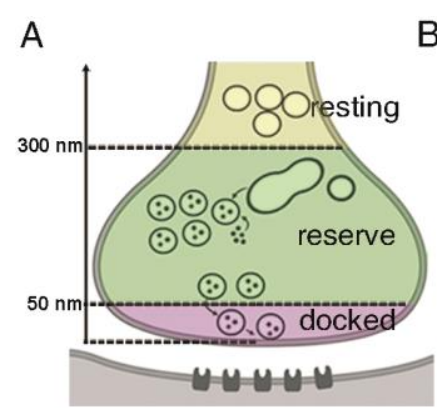

C

SVs density

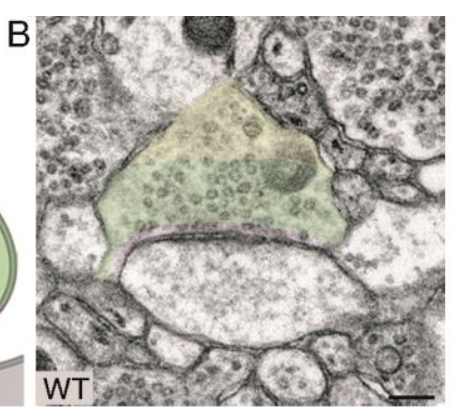

D

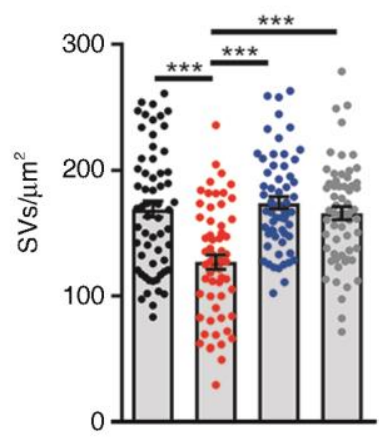

G

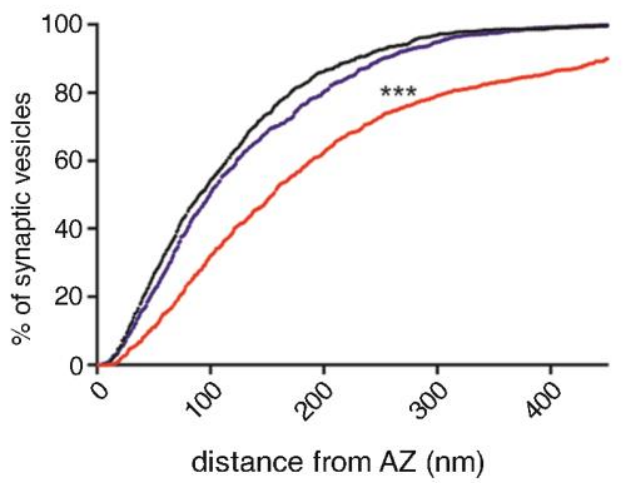

Synaptic area

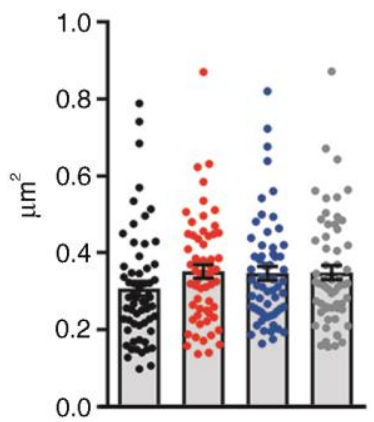

$\mathrm{H}$

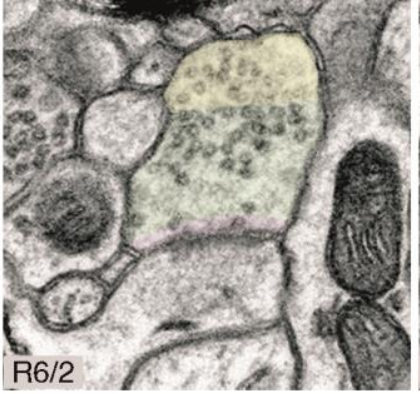

E

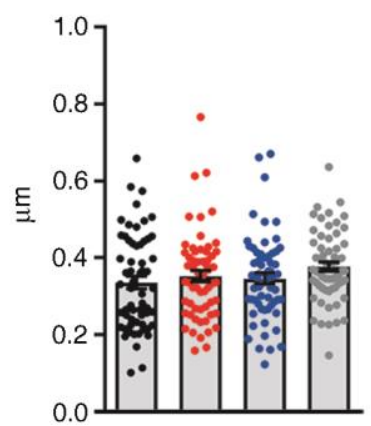

AZ length

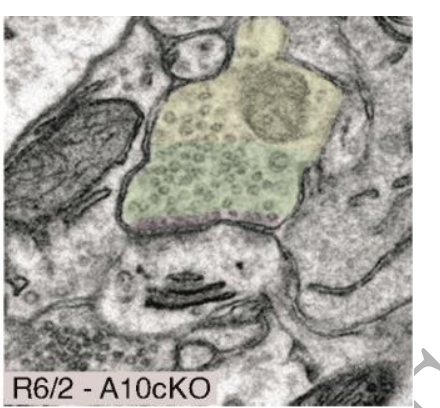

$\mathrm{F}$

SVs diameter

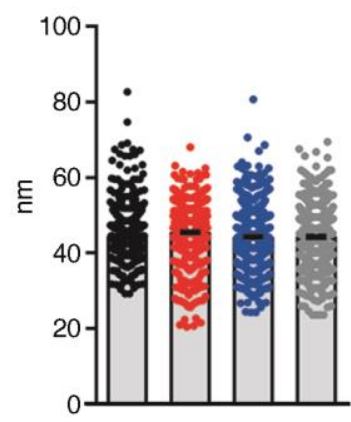

$\mathrm{J}$ Resting SVs

(G) Plot of the cumulative \% of SVs distribution within 0 to $500 \mathrm{~nm}$ from the AZ.

(H-J) Density of docked (H), reserve (1), and resting (J) synaptic vesicle pools in wild-type, R6/2, R6/2-A10cKO, and A10cKO mice. Each dot represents one synapse. We analyzed 3 mice/genotype and 60 synapses/genotype. 
Data are presented as mean $\pm \mathrm{SEM} ; * * \mathrm{P}<0.01, * * * \mathrm{P}<0.001$, ANOVA followed by Tukey’s multiple comparison test.

\section{Abbreviations}

A Disintegrin and metalloproteinase domain-containing protein 10: ADAM10

active zone (AZ)

adaptor related protein complex 2 subunit alpha 1: AP2A1

amyloid precursor protein: APP

bassoon: BSN

co-immunoprecipitation: co-IP

Contaminant Repository for Affinity Purification database: CRAPome

Data Depending Acquisition: DDA

discs large MAGUK scaffold protein 1: DLG1

dithiothreitol: DTT

ELKS/RAB6-Interacting/CAST Family Member 2: ERC2

false discovery rate: FDR

fold-change: FC

horseradish peroxidase: HRP

Huntingtin gene, human: HTT

Huntingtin protein, mouse and human: HTT

Huntington's Disease: HD

immunoaffinity purification-mass spectrometry: IP-MS

liquid chromatography with tandem mass spectrometry: LC-MS/MS

mature ADAM10: m-ADAM10

nanoscale liquid chromatography coupled to tandem mass spectrometry: nanoLC-MS/MS

N-cadherin: N-CAD

nectin cell adhesion molecule 1: NECTIN1

neuroligin 1: NLGN1 
phenylmethylsulfonyl fluoride: PMSF

piccolo: PCLO

polymerase chain reaction: PCR

postsynaptic density protein 95: (PSD-95)

postsynaptic density: PSD

proconvertase 7: PC7

sodium dodecyl sulfate: SDS

sodium dodecyl sulfate-polyacrylamide gel electrophoresis: SDS-PAGE

synapse-associated protein 97: SAP97

synaptic vesicles: SVs

synaptophysin: SYP

trichloroacetic acid: TCA

tetraspanin-15: TSPAN15

transmission electron microscopy: TEM 